\title{
Comparing serum concentration of spexin among patients with metabolic syndrome, healthy overweight/obese, and normal-weight individuals
}

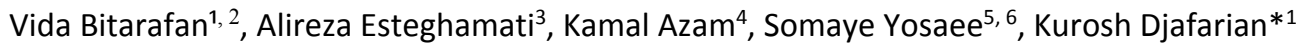 \\ Received: 9 Aug 2018 \\ Published: 6 Sep 2019
}

\section{Abstract}

Background: There are many factors related to etiology of metabolic syndrome (MetS) including obesity. Spexin, a peptide hormone released from adipose tissue, is the most down-regulated gene in obese, compared to non-obese adipose tissue. Hence, it potentially contributes to the progression and development of metabolic diseases. This study was designed to evaluate serum concentration of spexin in patients with MetS compared to weight-matched and normal-weight controls.

Methods: In this case-control study, 153 participants (51 per group) were collected from October 2014 to June 2016. The study groups were all matched for age and sex and included overweight/obese individuals with MetS and 2 control groups without MetS (including weight-matched and normal-weight participants). Body composition and serum concentration of spexin and leptin were measured.

Results: Serum leptin and spexin levels were significantly higher and lower, respectively, in normal-weight compared to overweight/obese groups with/without MetS $(p<0.02)$. No significant difference was observed in serum leptin and spexin concentrations between the overweight/obese groups with/without MetS ( $\mathrm{p} \geq 0.05$ ). Also, spexin, with cutoff value of 4.6 , had $78 \%$ sensitivity and $82 \%$ specificity for diagnosing overweight/obese from normal-weight participants. Spexin had $78 \%$ sensitivity and specificity, with cutoff value of 4.35, in diagnosing MetS participants from normal-weight group.

Conclusion: This study found no correlation between the circulating level of spexin and MetS development. Higher serum concentration of spexin in normal-weight adults compared to the obese participants illustrated the potential role of this novel peptide in obesity.

Keywords: Metabolic syndrome, Obesity, Spexin, Leptin

Conflicts of Interest: None declared

Funding: Tehran University of Medical Sciences

*This work has been published under CC BY-NC-SA 1.0 license.

Copyright $₫$ Iran University of Medical Sciences

Cite this article as: Bitarafan V, Esteghamati A, Azam K, Yosaee S, Djafarian K. Comparing serum concentration of spexin among patients with metabolic syndrome, healthy overweight/obese and normal-weight individuals. Med J Islam Repub Iran. 2019 (6 Sep);33:93. https://doi.org/10.47176/mjiri.33.93

\section{Introduction}

Metabolic syndrome (MetS) consists of elevated risk factors for heart disease, abdominal obesity, hypertension, glucose intolerance, and dyslipidemia (1). The other diagnostic factors are hypertension (HTN), high triglycerides (TG),

Corresponding author: Dr Kurosh Djafarian, kdjafarian@tums.ac.ir

1. Department of Clinical Nutrition, School of Nutritional Sciences and Dietetics, Tehran University of Medical Sciences, Tehran, Iran

2. Adelaide Medical School, Faculty of Health and Medical Sciences, University of Adelaide, Adelaide 5005, Australia

3. Endocrinology and Metabolism Research Center (EMRC), Vali-Asr Hospital, Tehran University of Medical Sciences, Tehran, Iran

4. Department of Epidemiology and Biostatistics, School of Public Health, Tehran University of Medical Sciences, Tehran, Iran

5. Department of Nutrition Sciences, School of Health, Larestan University of Medical Sciences, Larestan, Iran

6. Department of Nutrition Sciences, Emam Reza Teaching Hospital, Larestan University of Medical Sciences, Larestan, Iran and low high-density lipoprotein (HDL) (2). This syndrome has spread all over the world and is now known as a public health dilemma (3-6). Globally, $20 \%$ to $25 \%$ of adults already have MetS (7). It has been reported that MetS has extremely high prevalence in Iran. For example, the

\section{$\uparrow$ What is "already known" in this topic:}

Obesity plays an important role in MetS incidence. Spexin, as the most down-regulated gene in obese compared to non-obese adipose tissue, is potentially a protective agent against obesity development, but it's possible roles in relation to MetS are unknown.

\section{$\rightarrow$ What this article adds:}

The circulating level of spexin is not correlated with MetS development. Overweight/obese adults have lower serum spexin circulation compared to normal weight adults, which illustrates a novel role of spexin in regulating obesity. 
Tehran lipid and glucose study (7) has shown that MetS prevalence is $42 \%$ and $24 \%$ among Iranian women and men, respectively (7).

Obesity is known as the most important cause of MetS incidence (8). White adipose tissue (WAT) has a critical role in energy storage and also functions as an "endocrine organ" by secreting adipokines, which can effect energy homeostasis maintenance, appetite, satiety, glucose, and lipid metabolism by regulating insulin activity, energy metabolism, glucose storage, and long chain fatty acid (LCFA) uptake via both endocrine and paracrine mechanisms (9). Hormonal signals to the white adipose tissue, such as spexin, might underpin the MetS development (10-12). Comparing gene expression in non-obese versus obese human omental and subcutaneous fat confirmed that energy homeostasis-associated gene (Ch12:orf39) is the most down-regulated gene in obese white adipose tissue (12).

Spexin was discovered by Mirabeau et al in 2007 (13). It is encoded by the Ch12:orf39 gene that is expressed in different body tissues (14-16). Spexin plays a major role in obesity by regulating energy and appetite, controlling feeding behaviours, decreasing $\mathrm{O}_{2}$ consumption, $\mathrm{CO}_{2}$ production, respiratory exchange ratio (RER), energy expenditure (EE), and increasing locomotion, inhibiting LCFA uptake into adipocytes, suggesting spexin may be a key player in MetS development (17). In addition to its effects on weight regulation, spexin is related to central nervous system (CNS)-mediated control of salt and water balance and arterial blood pressure. Therefore, potentially contributes to the progression and development of metabolic diseases (18, 19).

Leptin, an adipocytokine, plays a critical role in regulation of food intake (20), energy expenditure (21), and body weight (22), hence leptin resistance is an early manifestation of MetS (23). Serum leptin levels in patients with MetS are higher than that those in healthy participants (24). Increased leptin level predicts MetS development independent of obesity (25). It seems that spexin and leptin may play antagonistic roles in regulation of body weight and adiposity, and due to their negative correlation in the serum of normal-weight participants and obese patients, low spexin and high leptin serum levels may contribute to MetS development $(13,17)$.

To date, spexin and leptin have not been studied in obese patients with MetS and obese patients without MetS or normal-weight individuals, thus their possible role in MetS is unknown. The aim of this study was to investigate the hypothesis that serum circulating level of spexin differs between patients with MetS and those age, sex, and BMI matched controls.

\section{Methods}

\section{Participants}

This was a case-control study with 51 cases and 102 controls in 2 groups. The differences in the mean levels of waist to hip ratio was expected to be at least $5 \%$ with the standard deviation of 0.14 in the case and control groups. Therefore, the desired power was set at 0.8 to detect a true effect (26). The cases were patients newly diagnosed with MetS (based on the ATPIII panel criteria (27), with the BMI of $\geq 25$ (body mass index), who had never received medications. They were recruited from patients who had referred to the Endocrinology Center of Tehran University of Medical Sciences. The control groups were age and gender matched to the cases, and consisted of 51 overweight/obese individuals without MetS and 51 normal-weight individuals selected from those attending the Endocrinology Center for routine medical care. BMI was matched between the 2 overweight/obese groups with/without MetS. Exclusion criteria for all 3 groups were pregnancy, lactation, suffering from any kind of cancer, liver disorder, kidney disorder, blood disorder, uncontrolled thyroid disease, and ischemic heart diseases, using medication for modifying serum lipids and glucose, sedative or hypnotic drug, antihistamine, immune system inhibitors and following any special diet under the supervision of a dietician, being a professional athlete, and smoking at least once a week.

\section{Anthropometric and general information}

The research protocol of the study was reviewed and approved by the scientific research committee of Tehran University of Medical Sciences. Demographic information was obtained after obtaining written informed consent from each participant. Participants were weighed and measured with light clothing and without shoes using a Seca ${ }^{\text {TM }}$ (Hanover, MD) portable scale. Also, body composition was assessed using BIA (bioelectrical impedance analysis) and a wall-mounted stadiometer. Waist circumference (WC) was measured using a flexible anthropometric tape midway between the iliac crest and lower rib margin. BMI was calculated by the ratio of weight $(\mathrm{kg})$ to height squared $\left(\mathrm{m}^{2}\right)$ ratio. Diastolic blood pressure (DBP) and systolic blood pressure (SBP) were measured using GAMMA oscillo-metric method after the patients rested for at least 10 minutes in relaxed and seated position. Measurement was repeated 2 times and the average was reported.

\section{Biochemical analyses}

Blood samples were collected after 8-12 hours of fasting and 20 minutes of supine rest. Venous blood was drawn into EDTA tubes and centrifuged at $4^{\circ} \mathrm{C}$ p; then, plasma was frozen at $-80^{\circ} \mathrm{C}$ until biochemical analyses were performed. Fasting blood sugar (FBS) was measured using an automated glucose oxidase method (Automatic Analyzer 2700, Olympus, Japan). Triglyceride (TG), serum total cholesterol (TC), low-density lipoprotein-cholesterol (LDLC), and high-density lipoprotein-cholesterol (HDL-C) were measured using enzymatic methods.

\section{Spexin and leptin}

The spexin serum concentration was measured by a commercial ELISA kit (Cat \# CSB-EL003071HU) purchased from Phoenix Pharmaceuticals (Burlingame, CA). The assay recognizes natural human and recombinant spexin. No significant interference or cross-reactivity was observed. Test range was $62.5-4000 \mathrm{pg} / \mathrm{mL}$. The sensitivity of the assay was $15.6 \mathrm{pg} / \mathrm{mL}$, and the interassay and intraassay coefficients of variation (CV) were less than $10 \%$ and $8 \%$, respectively. The enzyme-linked immune-sorbent assay (ELISA) was used to measure serum leptin levels using a 
Mediagnost Kit (Reutlingen/Germany). The assay was conducted according to the manufactures' instruction.

\section{Statistical analysis}

Data were represented with mean and standard deviation for quantitative variables and frequency and percentage for qualitative variables. The normality of the variables distribution was examined using one-sample KolmogorovSmirnov test. To compare the means of the normal distributed variables in the case and control groups, one-way analysis of variance (ANOVA) test was performed and nonparametric Kruskal-Wallis test was used to compare the distribution of the variables that were not normally distributed. In addition, to compare the means of the normal distributed variables within groups, Tukey post hoc test was used and Mann-Witney U test was performed for the variables that were not normally distributed. Also, Pearson correlation was used to evaluate the linear correlation between spexin and body composition indices. Moreover, multiple linear regression models were used to assess the association between spexin, leptin, and related factors. A stepwise backward elimination process was performed to construct the final model. At first, all variables with $p$-value of $<0.20$ were entered into the model; then, variables were eliminated according to the LRT (Likelihood Ratio Test) until no more variables could be removed by LRT. The receiver operator characteristic curve (ROC curves) was used to determine the appropriate cutoff point of spexin with highest sensitivity and specificity in diagnosing MetS. A 2-tailed $p$ value $<0.05$ was considered statistically significant for all analyses. Statistical Package for the Social Sciences (SPSS) software, version 24 (SPSS Inc., Chicago, IL, USA) was used to perform the analyses.

\section{Results \\ Participants}

MetS characteristics and general characteristics of study population are demonstrated in Table 1. Most participants in all groups were men. In terms of age, sex, and height status, no statistical significance was observed between the 3 groups (all $\mathrm{p}$ values were less than 0.05 ), indicating that the matching was successful. No significant difference was found between the first and second groups ( $\mathrm{p}=0.281)$. MetS components, including blood pressure, TG, FBS, and WC were significantly higher among MetS patients compared to both overweight/obese without MetS and normal-weight control participants, while HDL-C was the lowest in case patients $(\mathrm{p}<0.001)$.

\section{Body composition}

The mean of anthropometric measurements, including the total and regional body composition of the 3 groups, are displayed in Table 2. The overweight/obese groups with/without MetS had a significantly higher total and regional body fat and lower free fat mass compared to the normal-weight group $(\mathrm{p}<0.001)$, while no significant differences were observed for body free fat and fat mass between the MetS group and the overweight/obese without MetS group $(\mathrm{p}=0.939)$.

\section{Spexin and leptin}

The mean concentration of serum spexin was significantly higher in normal-weight participants $(9.86 \pm 5.21)$ compared to overweight/obese groups with MetS $(2.73 \pm 1.72)$ and without MetS $(2.59 \pm 1.82) \quad(p<0.001)$. However, the mean serum spexin concentration was not significantly different between the overweight/obese groups with/without MetS ( $\mathrm{p}=0.655)$ (Graph 1).

A significant difference was seen in leptin levels of overweight/obese patients with and without MetS groups and normal-weight participants $(p=0.037)$. Leptin levels were higher in patients with MetS $(14.06 \pm 12.4)$ compared to overweight/obese without MetS (11.2 \pm 9.3$)$ and normalweight without MetS (7.09 \pm 7.1$)$, respectively $(p=0.002)$ (Graph 1). In addition, the trend of spexin and leptin, according to the number of MetS components, are also shown in Graph 2.

Receiver operator characteristic curve (ROC curve) and area under the curve (AUC) for determining the ability of spexin in diagnosing obesity/overweight from normalweight participants are shown in Graph 3. The area under the ROC curve was 0.89 for spexin levels $(p<0.001)$. For the cutoff value of 4.6 , the sensitivity and specificity values of spexin levels were $78 \%$ and $82 \%$, respectively. ROC

Table 1. Characteristics of the participants

\begin{tabular}{|c|c|c|c|c|c|c|}
\hline \multicolumn{2}{|c|}{ Variables } & \multirow{2}{*}{$\begin{array}{c}\text { Overweight/obese } \\
\text { with MetS } \\
(\mathrm{n}=51)\end{array}$} & \multirow{2}{*}{$\begin{array}{c}\text { Overweight/obese with- } \\
\text { out MetS } \\
(\mathrm{n}=51)\end{array}$} & \multirow{2}{*}{$\begin{array}{c}\begin{array}{c}\text { Normal-weight without } \\
\text { MetS } \\
(\mathrm{n}=51)\end{array} \\
44(86.3)\end{array}$} & \multirow{2}{*}{$\begin{array}{c}\text { Total } \\
132 \\
\end{array}$} & \multirow[t]{2}{*}{$\begin{array}{l}\mathrm{p} \\
1\end{array}$} \\
\hline Gen- & Male, n (\%) & & & & & \\
\hline Age, $y$ & & $37.47 \pm 6.50$ & $36.94 \pm 6.4$ & $35.93 \pm 6.37$ & $36.78 \pm 6.40$ & 0.409 \\
\hline Body & & $92.50 \pm 13.32$ & $13.73 \pm 89.19$ & $6.80 \pm 69.13$ & $15.58 \pm 83.61$ & $<0.001$ \\
\hline Heigh & & $7.74 \pm 171.49$ & $7.03 \pm 172.26$ & $6.83 \pm 171.18$ & $7.18 \pm 171.64$ & 0.572 \\
\hline BMI, & & $31.43 \pm 3.72$ & $29.95 \pm 3.60$ & $23.65 \pm 1.43$ & $28.34 \pm 4.57$ & $<0.001 *$ \\
\hline Waist & erence, $\mathrm{cm}$ & 8.16105 .09 & 11.35100 .20 & $7.0 \pm 87.02$ & 11.7997 .44 & $<0.001$ \\
\hline Hip ci & ence, $\mathrm{cm}$ & $107.91 \pm 7.44$ & $106.65 \pm 6.23$ & $96.83 \pm 4.19$ & $103.77 \pm 7.83$ & $<0.001$ \\
\hline Clinic & blood pressure, $\mathrm{mmHg}$ & $11.76 \pm 121.20$ & $8.62 \pm 113.80$ & $12.22 \pm 111.37$ & $11.69 \pm 115.46$ & $<0.001$ \\
\hline $\begin{array}{l}\text { Clinic } \\
\mathrm{mmH}\end{array}$ & c blood pressure, & $9.12 \pm 80.80$ & $6.68 \pm 75.71$ & $8.38 \pm 74.14$ & $8.56 \pm 76.88$ & $<0.001$ \\
\hline FBS, & & $37.39 \pm 116.25$ & $20.40 \pm 97.80$ & $7.63 \pm 95.24$ & $103.10 \pm 26.49$ & $<0.001$ \\
\hline Trigly & $\mathrm{mmol} / \mathrm{L}$ & $203.60 \pm 266.3$ & $87.45 \pm 131.05$ & $67.87 \pm 117.56$ & $149.02 \pm 171.64$ & $<0.001$ \\
\hline HDL, & & 8.3550 .33 & 5.6154 .31 & 9.2054 .31 & $53.24 \pm 8.09$ & 0.010 \\
\hline
\end{tabular}


Table 2. Comparison of the total and regional body composition among case and control groups

\begin{tabular}{|c|c|c|c|c|}
\hline & $\begin{array}{c}\text { Overweight/obese with MetS } \\
(\mathrm{n}=51)\end{array}$ & $\begin{array}{l}\text { Overweight/obese without MetS } \\
(\mathrm{n}=51)\end{array}$ & $\begin{array}{c}\text { Normal-weight without MetS } \\
(\mathrm{n}=51)\end{array}$ & $\mathrm{p}$ \\
\hline & $\mathrm{SD} \pm$ Mean & $\mathrm{SD} \pm$ Mean & $\mathrm{SD} \pm$ Mean & \\
\hline Free fat mass $(\%)^{a / b}$ & $72.9 \pm 6.2$ & $74 \pm 6.6$ & $80.6 \pm 6$ & $<0.001$ \\
\hline Fat mass $(\%)^{a / b}$ & $27.1 \pm 6.2$ & $25.9 \pm 6.6$ & $19.4 \pm 6.1$ & $<0.001$ \\
\hline Trunk fat $(\%)^{a / b}$ & $28.9 \pm 5.4$ & $27.9 \pm 6.4$ & $20.6 \pm 6$ & $<0.001$ \\
\hline Right hand fat mass $(\%)^{a / b}$ & $26.1 \pm 8.4$ & $25.2 \pm 7.7$ & $18.5 \pm 6.2$ & 0.001 \\
\hline Left hand fat mass $(\%)^{a / b}$ & $26.9 \pm 8.5$ & $26.2 \pm 7.9$ & $19.1 \pm 6.6$ & $<0.001$ \\
\hline Right leg fat mass $(\%)^{a / b}$ & $24.2 \pm 7.9$ & $22.7 \pm 7.9$ & $17.4 \pm 7.2$ & $<0.001$ \\
\hline Left leg fat mass $(\%)^{a / b}$ & $24.7 \pm 8.9$ & $22.9 \pm 7.7$ & $17.6 \pm 7$ & $<0.001$ \\
\hline Leptin(ng/ml $)^{a / b}$ & $14.1 \pm 12.4$ & $11.2 \pm 9.3$ & $7.1 \pm 7.1$ & 0.002 \\
\hline $\operatorname{Spexin}(\mathrm{ng} / \mathrm{ml})^{a / b}$ & $2.7 \pm 1.72$ & $2.6 \pm 1.82$ & $9.8 \pm 5.21$ & $<0.001$ \\
\hline spexin/leptin ${ }^{a / b}$ & $0.2 \pm 0.09$ & $0.3 \pm 0.75$ & $1.7 \pm 0.73$ & $<0.001$ \\
\hline
\end{tabular}

Values are analyzed by one-way ANOVA

${ }^{a}$ Group 1 vs. Group 2 is significant; $\mathrm{p} \leq 0.05$

${ }^{\mathrm{b}}$ Group1 vs Group3 is significant; $\mathrm{p} \leq 0.05$

${ }^{\mathrm{c}} \mathrm{Group} 2$ vs. Group3 is significant; $\mathrm{p} \leq 0.05$

Table 3. Multiple linear regression for assessing an association between spexin and related factors

\begin{tabular}{|c|c|c|c|c|c|c|c|c|}
\hline \multirow[t]{2}{*}{ Variables } & \multicolumn{2}{|c|}{ Model $1^{\mathrm{a}}$} & \multicolumn{2}{|c|}{ Model $2^{\mathrm{b}}$} & \multicolumn{2}{|c|}{ Model $3^{c}$} & \multicolumn{2}{|c|}{ Model 4d } \\
\hline & $\mathrm{B}$ & $\mathrm{p}$ & $\mathrm{B}$ & $\mathrm{p}$ & $\mathrm{B}$ & $\mathrm{p}$ & $\mathrm{B}$ & $\mathrm{p}$ \\
\hline Leptin & -0.095 & 0.01 & -0.053 & 0.277 & 0.083 & 0.051 & 0.084 & 0.037 \\
\hline Age & -0.037 & 0.543 & & & & & & \\
\hline Gender & 4.94 & $<0.001$ & & & 9.372 & $<0.001$ & 7.164 & $<0.001$ \\
\hline Group 1 & -7.311 & $<0.001$ & -3.769 & $<0.001$ & -9.440 & $<0.001$ & -9.205 & $<0.001$ \\
\hline Group 2 & -7.095 & $<0.001$ & -3.532 & $<0.001$ & -10.922 & $<0.001$ & -10.744 & $<0.001$ \\
\hline Waist circumference & -0.144 & $<0.001$ & -0.126 & $<0.001$ & 0.042 & 0.327 & & \\
\hline BMI & -0.398 & $<0.001$ & -0.413 & $<0.001$ & 0.212 & 0.148 & 0.307 & 0.018 \\
\hline SBP & -0.136 & $<0.001$ & -0.111 & $<0.001$ & -0.079 & 0.010 & -0.070 & 0.001 \\
\hline DBP & -0.130 & 0.004 & -0.107 & 0.012 & 0.026 & 0.550 & & \\
\hline FBS & -0.036 & 0.014 & -0.032 & 0.024 & -0.004 & 0.627 & & \\
\hline TG & -0.007 & 0.009 & -0.006 & 0.029 & 0.001 & 0.635 & & \\
\hline HDL & 0.090 & 0.057 & 0.054 & 0.249 & -0.007 & 0.823 & & \\
\hline MetS component & -1.077 & $<0.001$ & -1.071 & $<0.001$ & 0.685 & 0.40 & 0.794 & 0.005 \\
\hline Basal metabolic rate & -0.002 & $<0.001$ & -0.002 & $<0.001$ & -0.003 & 0.270 & & \\
\hline Fat mass & -0.104 & 0.027 & -0.173 & $<0.001$ & -0.831 & 0.001 & -0.833 & 0.001 \\
\hline Fat free mass & 0.222 & $<0.001$ & 0.214 & $<0.001$ & 0.451 & 0.226 & & \\
\hline Trunk fat & -0.237 & 0.003 & -0.262 & 0.001 & 1.379 & $<0.001$ & 1.346 & $<0.001$ \\
\hline
\end{tabular}

Study groups include control group, normal-weight without MeS (Group 1), overweight/obese without MetS (Group 2), overweight/obese with MetS.

${ }^{a}$ Unadjusted effect of variables

${ }^{\mathrm{b}}$ Adjusted for demographic variables such as gender, age, marital status, and educational level

${ }^{c}$ Adjusted model (all variables, with p-value $<0.2$, entered the model such as leptin, age, gender, group1, group2, waist circumference, BMI, SBP, DBP, FBS, TG, HDL,

MetS component, BMR, fat mass, fat free mass, trunk fat).

${ }^{\mathrm{d}}$ Multivariable backward linear regression model.

curve was also used to determine the ability of spexin in diagnosing MetS from normal-weight participants (Graph 4). The area under the ROC curve was 0.87 for spexin levels $(\mathrm{p}<0.001)$. For the cutoff value of 4.35 , the sensitivity and specificity values of spexin levels were 0.78 .

\section{Relationship between Spexin and related factors}

There was a negative linear association between serum

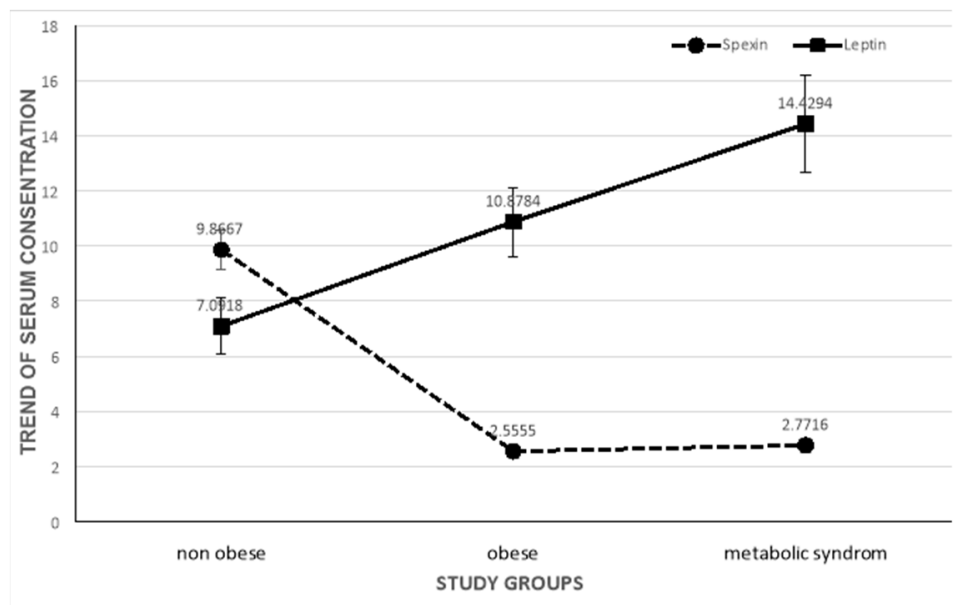

Error bars represent 1 +/- SEM (Standard Error of Mean)

Graph 1. Trend of spexin and leptin according to study groups 


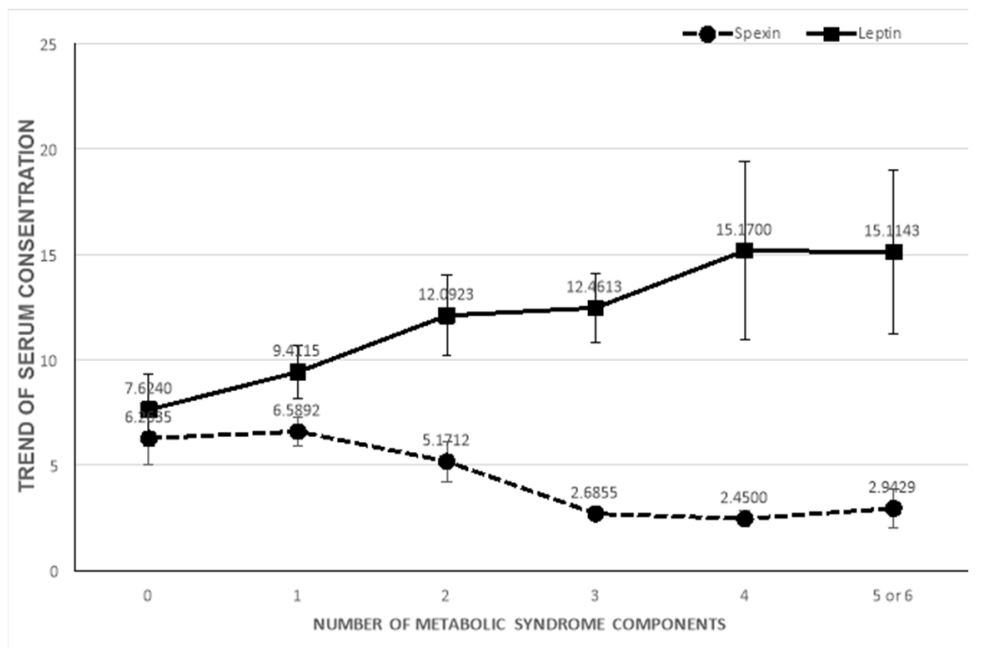

Error bars represent $1+/$ - SEM

Graph 2. Trend of spexin and leptin according to number of metabolic syndrome components

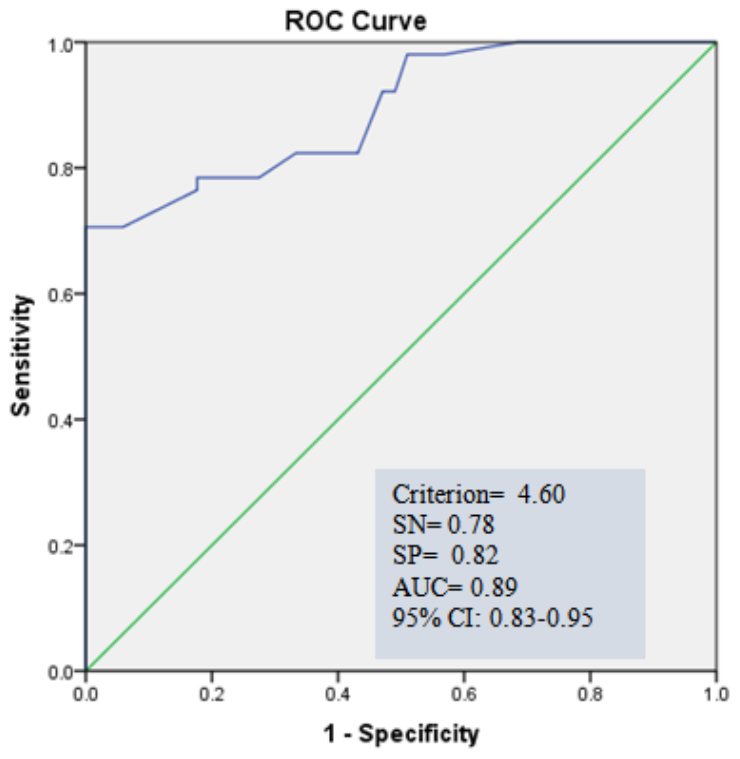

Diagonal segments are produced by ties.

Graph 3. ROC curve for spexin for diagnosing non metabolic syndrome in overweight/obese from normal-weight group

spexin concentration with age, serum leptin levels, waist circumference, BMI, SBP, DBP, FBS, TG, basal metabolic rate, fat mass, and trunk fat (all $\mathrm{P}<0.05$ ), but a trend of positive linear association with $\operatorname{HDL}(\mathrm{P}=0.057)$ and a positive linear association with fat free mass $(\mathrm{P}<0.001)$ Table 3 .

\section{Discussion}

In the present study, the concentrations of serum spexin and leptin and body composition (fat mass, fat-free mass) were compared among patients with MetS and 2 control groups. This study provided evidence that suggests a negative correlation for serum spexin and a positive correlation for serum leptin levels and occurrence of obesity, but no significant link between circulating spexin and leptin levels and MetS.

In this study, although the obese participants without

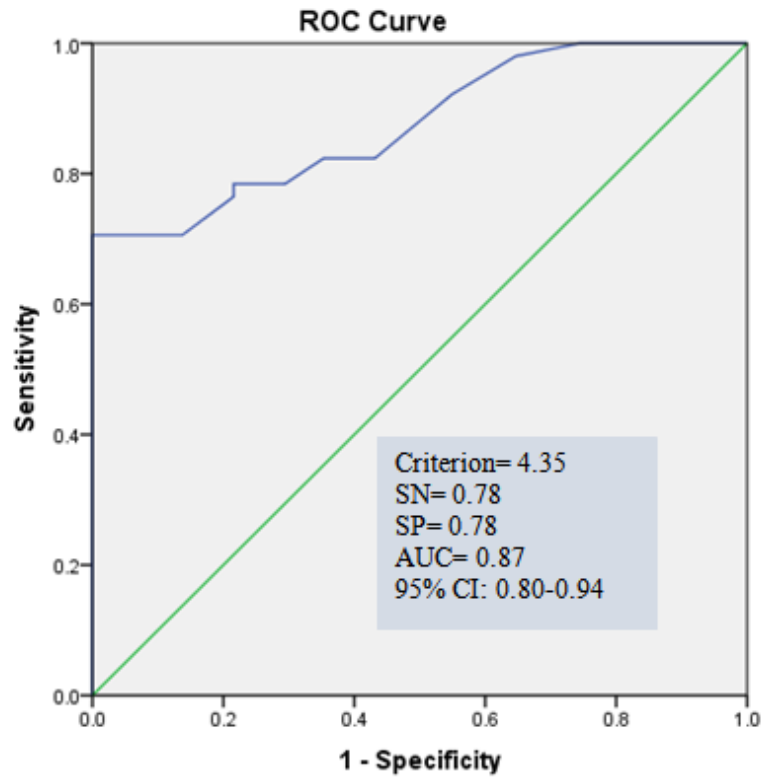

Diagonal segments are produced by ties.

Graph 4. ROC curve for spexin for diagnosing metabolic syndrome from normal-weight group

MetS had an unhealthy serum leptin level and body composition, in terms of MetS components, including high FBS, HDL, TG, and blood pressure, they were in a healthy status. This suggests that the difference in leptin concentration and body compositions may not be a diagnostic criterion for a normal metabolic status in healthy obese individuals and other factors, including endocrine factors, may affect this disorder. Limited data are available on the association between spexin levels and metabolic disorders (16, 17).

In the present study, lower serum concentration of spexin $(4.60 \mathrm{ng} / \mathrm{mL})$ in the obese groups compared to the normalweight participants suggested that the serum concentration of spexin may play a potential role in obesity treatment and prevention. Our data supports the previous finding (13) that showed approximately $10 \%$ reduction in the mean of serum 
spexin concentration of obese patients compared to normalweight participants $(1.1 \pm 0.7$ vs $11.6 \pm 1.3 \mathrm{ng} / \mathrm{mL}$; obese vs nonobese) (17). These correlational studies could not find whether low spexin levels cause the increase in obesity or whether obesity reduces spexin levels. Previous studies illustrated that spexin may affect obesity regulation by different mechanisms, including increasing locomotors activity and RER, decreasing carbohydrate consumption and increasing lipid oxidation, decreasing lipogenesis, and changing feeding behavior by decreasing meal consumption (17). In goldfish, spexin stimulated satiety as a result of central alteration (18) and controlled blood pressure through the central mediators (16).

Obesity is considered as the most critical risk factor of MetS (8). Considering the protective role of spexin in obesity, a hypothesis is proposed that spexin may be an important defense mechanism against developing MetS in patients with obesity. If serum spexin does not remain higher than $9.86 \pm 5.21 \mathrm{ng} / \mathrm{mL}$, MetS may develop along with obesity. To test this hypothesis, spexin was measured in healthy overweight/obese participants and in those who were suffering from MetS. The findings of this study showed no significant difference in the serum spexin concentrations among both overweight and obese groups, indicating that the simultaneous presence of obesity and MetS compared to the mere presence of obesity is not associated with reduced levels of spexin and that serum spexin levels are not directly correlated with MetS incidence in overweight/obese participants. Future studies with longitudinal design are needed to observe the changes of spexin over time, which coincide with the development of obesity and elements of the MetS, to clarify the potential role of spexin in MetS.

Unusual body composition in obese patients is one of the important reasons of metabolic disorders (28). Findings of this study, for the first time, suggest that there is a negative and positive correlation between fat mass index and free fat mass index, respectively, with serum spexin level. In contrast, there is no difference between overweight/obese groups in serum spexin concentration and body composition components. This outcome suggests that measuring serum spexin along with body composition may provide a better evaluation for obesity.

\section{Conclusion}

This study indicated that the serum spexin concentration is not correlated with MetS. However, the lower serum concentration of spexin in normal-weight adults, compared to that in obese counterparts, identifies it as a potentially protective agent against obesity development. If this casual link is correct, then, administration of spexin or pharmaceuticals that promote its production may be a novel approach for prevention or treatment of obesity.

\section{Acknowledgements}

This project was supported by the grant from Tehran University of Medical Sciences (grant number: 26504-161-0293). The research group would like to thank all participants of the study.

\section{Conflict of interest}

The funding organization had no participation in the study design, data collection, analysis and interpretation, or in manuscript writing. Authors declare no potential conflicts of interests with respect to the research, authorship, and/or publication of this article.

\section{References}

1. Kim GW, Park HJ, Kim HS, Kim SH, Ko HC, Kim BS, et al. Analysis of cardiovascular risk factors and metabolic syndrome in korean patients with psoriasis. Ann Dermatol. 2012;24(1):11-5.

2. Alberti KG, Eckel RH, Grundy SM, Zimmet PZ, Cleeman JI, Donato $\mathrm{KA}$, et al. Harmonizing the metabolic syndrome: a joint interim statement of the International Diabetes Federation Task Force on Epidemiology and Prevention; National Heart, Lung, and Blood Institute; American Heart Association; World Heart Federation; International Atherosclerosis Society; and International Association for the Study of Obesity. Circulation. 2009;120(16):1640-5.

3. Lakka HM, Laaksonen DE, Lakka TA, Niskanen LK, Kumpusalo E, Tuomilehto J, et al. The metabolic syndrome and total and cardiovascular disease mortality in middle-aged men. JAMA. 2002;288(21):2709-16.

4. Lorenzo C, Williams K, Hunt KJ, Haffner SM. Trend in the prevalence of the metabolic syndrome and its impact on cardiovascular disease incidence: the San Antonio Heart Study. Diabetes Care. 2006;29(3):625-30.

5. Alexander CM, Landsman PB, Teutsch SM, Haffner SM. NCEPdefined metabolic syndrome, diabetes, and prevalence of coronary heart disease among NHANES III participants age 50 years and older. Diabetes. 2003;52(5):1210-4.

6. Bonora E, Kiechl S, Willeit J, Oberhollenzer F, Egger G, Bonadonna $\mathrm{RC}$, et al. Carotid atherosclerosis and coronary heart disease in the metabolic syndrome: prospective data from the Bruneck study. Diabetes care. 2003;26(4):1251-7.

7. Saberi HR, Moravveji AR, Fakharian E, Kashani MM, Dehdashti AR. Prevalence of metabolic syndrome in bus and truck drivers in Kashan, Iran. Diabetol Metab Syndr. 2011;3(1):8.

8. Gregor MF, Hotamisligil GS. Inflammatory mechanisms in obesity. Annu. Rev. Immunol. 2011;29:415-45.

9. Lago F, Gómez R, Gómez-Reino JJ, Dieguez C, Gualillo O. Adipokines as novel modulators of lipid metabolism. Trends Biochem. Sci. 2009;34(10):500-10.

10. Mirza S, Qu HQ, Li Q, Martinez PJ, Rentfro AR, McCormick JB, et al. Adiponectin/leptin ratio and metabolic syndrome in a Mexican American population. Clin Invest Med. 2011;34(5):E290.

11. Butler AA, Tam CS, Stanhope KL, Wolfe BM, Ali MR, O'keeffe M, et al. Low circulating adropin concentrations with obesity and aging correlate with risk factors for metabolic disease and increase after gastric bypass surgery in humans. J. Clin. Endocrinol. Metab. 2012;97(10):3783-91.

12. Walewski JL, Ge F, Gagner M, Inabnet WB, Pomp A, Branch AD, et al. Adipocyte accumulation of long-chain fatty acids in obesity is multifactorial, resulting from increased fatty acid uptake and decreased activity of genes involved in fat utilization. Obes Surg. 2010;20(1):93107.

13. Mirabeau O, Severini C, Audero E, Gascuel O, Possenti R, Birney E, et al. Identification of novel peptide hormones in the human proteome by hidden Markov model screening. Genome Res. 2007;17(3):320-7.

14. Porzionato A, Rucinski M, Macchi V, Stecco C, Malendowicz LK, De Caro R. Spexin expression in normal rat tissues. J. Histochem. Cytochem. 2010;58(9):825-37.

15. Liu Y, Li S, Qi X, Zhou W, Liu X, Lin H, et al. A novel neuropeptide in suppressing luteinizing hormone release in goldfish, Carassius auratus. Mol. Cell. Endocrinol. 2013;374(1):65-72.

16. Toll L, Khroyan TV, Sonmez K, Ozawa A, Lindberg I, McLaughlin $\mathrm{JP}$, et al. Peptides derived from the prohormone proNPQ/spexin are potent central modulators of cardiovascular and renal function and nociception. FASEB J. 2012;26(2):947-54.

17. Walewski JL, Ge F, Lobdell H, Levin N, Schwartz GJ, Vasselli JR, et al. Spexin is a novel human peptide that reduces adipocyte uptake of long chain fatty acids and causes weight loss in rodents with dietinduced obesity. Obesity. 2014;22(7):1643-52. 
18. Wong MK, Sze KH, Chen T, Cho CK, Law HC, Chu IK, et al. Goldfish spexin: solution structure and novel function as a satiety factor in feeding control. Am. J. Physiol. Endocrinol. Metab. 2013;305(3):E348-E66.

19. Kumar S, Hossain J, Nader N, Aguirre R, Sriram S, Balagopal PB. Decreased Circulating Levels of Spexin in Obese Children. J. Clin. Endocrinol. Metab. 2016;101(7):2931-6.

20. Mantzoros CS, Frederich RC, Flier JS, Qu D, Susulic VS, Lowell BB, et al. Activation of $\beta 3$ adrenergic receptors suppresses leptin expression and mediates a leptin-independent inhibition of food intake in mice. Diabetes. 1996;45(7):909-14.

21. Stehling O, Doring H, Ertl J, Preibisch G, Schmidt I. Leptin reduces juvenile fat stores by altering the circadian cycle of energy expenditure. Am. J. Physiol. Regul. Integr. Comp. Physiol. 1996;271(6):R1770-R4.

22. Mantzoros CS, Magkos F, Brinkoetter M, Sienkiewicz E, Dardeno TA, Kim SY, et al. Leptin in human physiology and pathophysiology. Am. J. Physiol. Endocrinol. Metab. 2011;301(4):E567-E84.

23. Zimmet P, Boyko E, Collier G, Courten Md. Etiology of the metabolic syndrome: potential role of insulin resistance, leptin resistance, and other players. Ann. N. Y. Acad. Sci. 1999;892(1):25-44.

24. Özçelik E, Uslu S, Kebapçı N, Kara M, Dokumacıŏglu A, Musmul A. Interrelations of serum leptin levels with adrenocorticotropic hormone, basal cortisol and dehydroepiandrosterone sulphate levels in patients with metabolic syndrome. Diabetes Metab Syndr. 2010;4(1):13-7.

25. Franks PW, Brage S, Luan Ja, Ekelund U, Rahman M, Farooqi IS, et al. Leptin predicts a worsening of the features of the metabolic syndrome independently of obesity. Obes. Res. 2005;13(8):1476-84.

26. Chen SJ, Yen CH, Huang YC, Lee BJ, Hsia S, Lin PT. Relationships between inflammation, adiponectin, and oxidative stress in metabolic syndrome. PLoS ONE. 2012;7(9):e45693.

27. Expert Panel on Detection E. Executive summary of the third report of the National Cholesterol Education Program (NCEP) expert panel on Detection, Evaluation, and Treatment of high blood cholesterol in adults (Adult Treatment Panel III). JAMA. 2001;285(19):2486.

28. Alberti KG, Zimmet P, Shaw J. Metabolic syndrome--a new worldwide definition. A Consensus Statement from the International Diabetes Federation. Diabet. Med. 2006;23(5):469-80. 